\title{
Avaliação da presença de tags intratubular na região apical de dentes anteriores unirradiculares, variando a técnica de manipulação do cimento resilon
}

\section{Evaluation ofthe presence of intratubular tags in the apical region of single- rooted anterior teeth, varying the technique of resilion cement handling}

\author{
1 Adriana Marques Nunes adrianaju@bol.com.br \\ 2 Rielson José Alves Cardoso \\ 3 Leonardo dos Santos Barroso \\ 3 Rosy de Oliveira Nardy Melo \\ ${ }^{4}$ Gesinete Gonçalves Pinto Klippel
}

\footnotetext{
1 Cirurgiã-dentista, Especialista e Mestre em Endodontia, São Leopoldo Mandic, Campinas/SP, Especialista em Ortodontia (IEPCCruzeiro/SP), Docente da Graduação e Pós-graduação, UniFOA.

2 Cirurgião-dentista, Coordenador do Curso de Mestrado em Endodontia na São Leopoldo Mandic, Campinas.

3 Cirurgião-dentista, Especialista e Mestre em Endodontia; Docente da Graduação e Pós-graduação, UniFOA.

4 Cirurgiã-dentista, Especialista em Endodontia; Docente Voluntária da Graduação e Pós-graduação, UniFOA.
}

\section{RESUMO}

Com o objetivo de avaliar a presença de tags do cimento de Resilon nos túbulos dentinários da região apical, variando a técnica de manipulação, foram selecionados vinte dentes unirradiculares humanos que tiveram suas coroas seccionadas no limite amelo-cementário. O preparo do canal foi realizado com três instrumentos além do diâmetro anatômico apical, com o uso das substâncias químicas hipoclorito de sódio a 2,5\%, endo-PTC e EDTA-T com irrigação passiva ultrassônica. Em seguida, os dentes foram divididos em dois grupos, sendo que o cimento para o grupo controle foi preparado com a ponta "automix" e o grupo espatulado foi acrescido de manipulação manual, ambos obturados com a técnica de termoplastificação do material obturador. Os dentes foram preparados para a análise na microscopia eletrônica de varredura, e para avaliar os resultados, as fotomicrografias foram qualificadas como amostras com presença de tags e amostras sem presença de tags. Quando avaliada a presença dos prolongamentos resinosos nas diferentes amostras nos resultados,pode-se verificar que, em ambos os grupos, o número foi o mesmo: cinco amostras apresentaram $(55,6 \%)$ e quatro não $(44,4 \%)$, tanto no grupo controle, como no grupo espatulado. Conclui-se, frente à metodologia utilizada, que os grupos tiveram o mesmo perfil de presença de tags produzidos, quando acrescida a espatulação manual do cimento preparado com a ponta "automix".

\section{Palavras-chave}

Resilon; tags; manipulação cimento; obturação; penetração de cimento.

\begin{abstract}
With the objective of evaluating the presence of tags formed with Resilon cement in dentinal tubules of the apical region, varying the manipulation technique, twenty singlerooted human teeth were selected and had their crowns sectioned in the amelo-cement limit. The canal preparation was performed with three instruments beyond the apical anatomic diameter, with the use of the chemical substances sodium hypochlorite $2.5 \%$, endo PTC and EDTA-T, with passive ultrasonic irrigation. Then the teeth were divided in two groups, the cement in control group being prepared with automix tip whereas the tooled group had added manual manipulation, both filled with the technique of termoplastification of the filling material. The teeth were prepared for analysis by scanning electron microscopy, and to evaluate the results, the photomicrographs were classified as samples with presence of tags and samples without the presence of tags. When the presence of resinous extensions was evaluated in the different samples in the results, it could be seen that both groups had the same number. five samples showed (55,6\%) and four not $(44,4 \%)$, both in the control group and the tooled group. It was concluded that, with the methodology used, both groups had the same profile regarding the presence of tags produced when added manual tooling cement prepared with the "automix" tip.
\end{abstract}

\section{Keywords}

Resilon; tags; manipulation sealer; obturation; sealer penetration.

\section{Como você deve citar?}

NUNES, Adriana Marques et al. Avaliação da presença de tags intratubular na região apical de dentes anteriores unirradiculares, variando a técnica de manipulação do cimento resilon. Cadernos UniFOA, Volta Redonda, n. 31, p. 103-112, ago. 2016. 
Avaliação da presença de tags intratubular na região apical de dentes anteriores unirradiculares, variando a técnica de manipulação do cimento resilon

\section{INTRODUÇÃo}

A obturação do sistema de canais radiculares é mais uma fase importantíssima para o sucesso do tratamento endodôntico, devendo este preencher todo o canal radicular anteriormente acessado, preparado e irrigado, a fim de permitir o selamento tridimensional.

O selamento produzido pela obturação do canal não impede a microinfiltração de bactérias, podendo levar ao insucesso do tratamento endodôntico (SHIPPER et al., 2004), porém os prolongamentos de cimento no interior da dentina, denominados de tags, melhora a interface material obturador/dentina, proporcionando melhores resultados na avaliação de microinfiltração (SHIPPER et al.,2004; APTEKAR, GINNAN, 2006), e também sua presença física e propriedade antimicrobiana, podendo eliminar ou inibir o crescimento do microorganismo no sistema de canais radiculares (SIQUEIRA et al., 2000; SALEH et al., 2004).

O material obturador denominado Epiphany ${ }^{\circledR}$ (PentronClinical Technologies, Wallingford, CT, EUA) consiste num sistema com cones de poliéster, um polímero sintético termoplástico com propriedades semelhantes à guta-percha, que é utilizado em conjunto com um primer e um cimento resinoso dual denominado Resilon, que possui uma ponta de mistura de componentes "automix", não sendo necessária a espatulação, segundo o fabricante. Esse sistema apresenta uma intenção de interação química que leva à formação de um monobloco de resina, representado por cimento e cone, que se adere à parede dentinária em toda a extensão do canal radicular, com uma penetração do cimento no espaço intratubular dentinário (SHIPPER et al.,2004; PATEL et al., 2007).

Devido ao cimento não apresentar um aspecto homogêneo após a sua colocação com a ponta "automix", avaliou-se a formação de tags intratubular produzidos, variando a técnica de manipulação do cimento Resilon, acrescendo espatulação manual.

\section{MATERIAIS E MÉTODOS}

Após aprovação pelo Comitê de Ética em Pesquisa ( $n^{\circ}$ 2008/0107), foram utilizados 20 dentes unirradiculares humanos completamente formados, com raízes retas e canal único, sem reabsorção interna e/ou externa apical, fornecidos, por doação, pelo Banco de Dentes do Centro de Pesquisas São Leopoldo Mandic, Campus Campinas-SP, armazenados em soro fisiológico.

Para dissolução de eventuais restos orgânicos, os dentes foram armazenados em hipoclorito de sódio a 2,5\%, por um período de 30 minutos. As coroas foram seccionadas no limite amelo-cementário, determinando um comprimento radicular de, no mínimo, $14 \mathrm{~mm}$.

O preparo químico cirúrgico seguiu os princípios de preparo no sentido coroa-ápice $1 \mathrm{~mm}$ aquém do forame apical, como segue: inicialmente, para o preparo cervical e médio, as brocas Gates Glidden $\mathrm{n}^{\circ}$ 3, 2, 1 foram utilizadas em ordem decrescente. Após, utilizou-se a broca LAAxess, para complementar o preparo cervical radicular. Após realizar a detecção do diâmetro anatômico apical, com auxílio de limas manuais do tipo $\mathrm{K}$, o preparo apical foi realizado com a técnica seriada, sendo o batente apical confeccionado com diâmetro de três limas acima do diâmetro anatômico. A irrigação com hipoclorito de sódio a $1 \%$ em quantidade de $5 \mathrm{ml}$ foi intercalada a cada instrumento manual a $2 \mathrm{~mm}$ do comprimento real de trabalho. Usou- se, também, Endo PTC como substância química auxiliar, ativado pelo hipoclorito de sódio a 1\%. A patência foraminal foi efetuada durante o preparo do canal radicular, até o último instrumento, realizada com instrumento tipo $\mathrm{K} \# 8$. 
Ao final, $5 \mathrm{ml}$ de EDTA a $17 \%$ foi utilizado como irrigante, deixando o canal preenchido por $3 \mathrm{~min}$ e uma lima \#15 inserida até o comprimento de trabalho e executado a PUI na potência 3 do ultrassom por $1 \mathrm{~min}$, afım de promover a remoção do smear layer. Posteriormente, realizou-se uma nova irrigação com $5 \mathrm{ml}$ de hipoclorito de sódio a 1,0\%, seguida de $10 \mathrm{ml}$ de soro fisiológico, para remover qualquer influência da substância irrigadora sobre o cimento obturador.

Os canais radiculares tiveram seu excesso de soro fisiológico removido por aspiração com a ponta Capilaritip ${ }^{\circledast}$, complementado por um cone de papel absorvente estéril de calibre correspondente ao diâmetro anatômico inicial, a fim de não remover por completo a umidade do canal,necessário ao cimento hidrofílico.

Após o preparo químico-cirúrgico os espécimes, foram distribuídos aleatoriamente em dois grupos, contendo 10 espécimes cada, conforme a técnica de manipulação do cimento endodôntico Epiphany ${ }^{\boxplus}$, sendo: Grupo espatulação (GE): manipulação "automix" acrescida de espatulação manual numa placa de vidro com uma espátula $\mathrm{n}^{\circ} 24$, por 10 segundos. Grupo controle (GC): manipulação conforme orientação do fabricante com a ponta "automix".

Todos os espécimes dos grupos experimentais foram obturados com cones de poliéster do próprio sistema Epiphany ${ }^{\circledR}$ pela técnica de termocompactação vertical, preconizada por Schilder (1967).

O primer autocondicionante foi aplicado com o auxílio de um cone de papel, sendo colocada uma gota no cone já posicionado dentro do canal radicular. 0 excesso foi removido com outro cone de papel seco. Após 30 segundos da aplicação do primer, o cimento foi introduzido, primeiramente, com lentulo e, depois, com o cone principal, pincelando-se todas as paredes do canal. Após a obturação dos canais radiculares, foi realizada a fotopolimerização por um minuto. 0 espaço de $2 \mathrm{~mm}$ deixado foi preenchido com cimento de ionômero de vidro restaurador, a fim de se promover o selamento provisório. Após obturação, os dentes foram armazenados em uma estufa a $37^{\circ} \mathrm{C}$ em umidade relativa de $100 \%$, durante 7 dias.

Para observação da parede dentino/pulpar, sulcos longitudinais nas faces mesial e distal das raízes foram feitos com disco metálico em micromotor de baixa rotação, com refrigeração abundante de água, porém sem expor o material obturador no interior dos canais radiculares, para não prejudicar os tags. Em seguida, realizou- se a clivagem das raízes, utilizando-se o instrumento lecron e fórceps $\mathrm{n}^{0}$ 151. Após, foi realizado o seccionamento do terço apical, contando, aproximadamente $5 \mathrm{~mm}$ do ápice e foi removido o material obturador manualmente. Os dois fragmentos da raiz foram analisados na região interna do canal radicular.

As amostras selecionadas passaram por um processo de desmineralização superficial, com aplicação de ácido fosfórico $37 \%$ por 1 minuto em toda dentina do canal radicular e, posteriormente, removido com soro fisiológico, expondo os túbulos dentinários que haveriam de possuir o cimento endodôntico no seu interior, como sugere o trabalho de Tay et al. (2005).

A seguir, as amostras foram submersas em placa petri, contendo hipoclorito de sódio a $5 \%$ para promover a limpeza de possíveis poeiras e óleo da clivagem e matéria orgânica e, após 10 minutos, foram irrigadas abundantemente com soro fisiológico. Posteriormente, as amostras foram levadas à estufa em $70^{\circ} \mathrm{C}$ por 10 minutos para desidratação, conforme apresentado nos trabalhos de Kouvas et al. (1998) e Tayet al.(2005). As amostras foram fixadas em plataformas especiais denominadas stubs, com auxílio de fita adesiva dupla face de carbono, para receberem o recobrimento de ouro em pó por um metalizador apropriado, cuja espessura é, em geral, da ordem de 100-200 . 
Avaliação da presença de tags intratubular na região apical de dentes anteriores unirradiculares, variando a técnica de manipulação do cimento resilon

A análise foi realizada por Microscópio Eletrônico de Varredura, visualizando-se a superfície interna da parede do canal radicular. Fez-se uma análise qualitativa com um aumento de 1000X das áreas com maior número de penetração do cimento nos túbulos dentinários do terço apical dos dois fragmentos radiculares. De cada amostra, foram obtidas duas fotomicrografias e escolhida a considerada melhor para a análise qualitativa. Todo o comprimento do fragmento radicular apical foi avaliado. As áreas com erosão, irregularidades presentes e que não foram possíveis visualizar com exatidão os tags foram descartadas da análise. As fotomicrografias realizadas para a avaliação mostraram os túbulos de vista frontal, sendo correspondente ao restante da área apical.

Para a análise qualitativa, as imagens foram avaliadas como contendo tags, ausência de tag se as imagens, quando o cimento estava com aspecto granular sem a formação de tags, foram classificadas como ausência de tags (Figura 01).

Figura 1 - Fotomicrografias ilustrando o critério de avaliação da analise qualitativa.

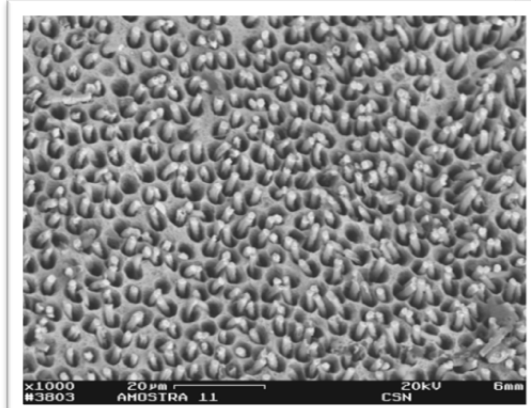

Com presença de tags

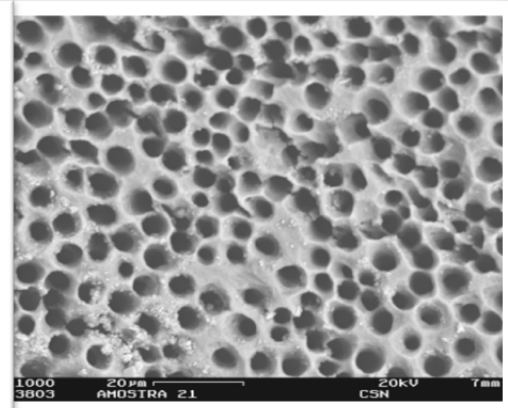

Sem presença de tags

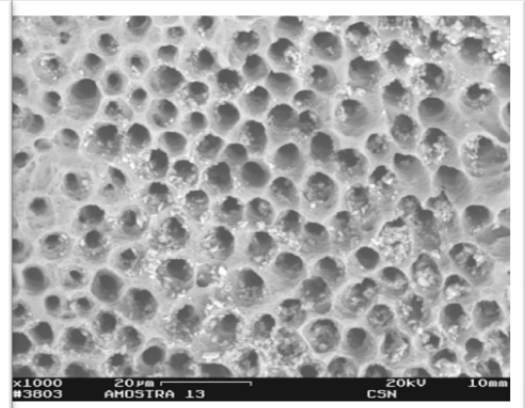

Sem presença de tags

Fonte: dos autores

\section{RESULTADOS}

As amostras seis e dezessete foram descartadas, pois, na clivagem, as avaliações ficaram prejudicadas. Portanto, nove espécimes de cada grupo foram avaliados. As dezoito amostras presentes foram observadas qualitativamente, se houve ou não presença de penetração intratubular do cimento endodôntico, formando tags de cimento (figura 4). Quando se avaliou a simples presença dos prolongamentos resinosos nas diferentes amostras, pode-se observar que, em ambos os grupos, esse número foi o mesmo, sendo cinco amostras com presença de tags (55,6\%), uma com ausência de tag se três foram descartadas, incluindo na contagem de amostras sem tags $(44,4 \%)$, tanto no grupo controle (figura 2), como no grupo espatulado (figura 3). Os dados globais dos grupos para a análise quantitativa das fotomicrografias da microscopia eletrônica de varredura foram inseridos na tabela 1, e avaliados estatisticamente. 
Adriana Marques Nunes / Rielson José Alves Cardoso / Leonardo dos Santos Barroso Rosy de Oliveira Nardy Melo I Gesinete Gonçalves Pinto Klippel

Tabela 1 - Tabela com avaliação da presença ou ausência de tags.

\begin{tabular}{|c|c|c|c|c|c|c|c|}
\hline Amostra & Com tags & Sem tags & Descartado & Amostra & Com tags & Sem tags & Descartado \\
\hline 1.1 & & $x$ & & 2.1 & $x$ & & \\
\hline 1.2 & $x$ & & & 2.2 & & & $x$ \\
\hline 1.3 & & & $x$ & 2.3 & & & $x$ \\
\hline 1.4 & & & $x$ & 2.4 & $x$ & & \\
\hline 1.5 & & & $x$ & 2.5 & $x$ & & \\
\hline 1.6 & $x$ & & & 2.6 & & & $x$ \\
\hline 1.7 & $x$ & & & 2.7 & $x$ & & \\
\hline 1.8 & $x$ & & & 2.8 & $x$ & & \\
\hline 1.9 & $x$ & & & 2.9 & & $x$ & \\
\hline
\end{tabular}

Fonte: dos autores

Figura 2 - Porcentagem de amostras com presença ou ausência de tags do grupo controle.

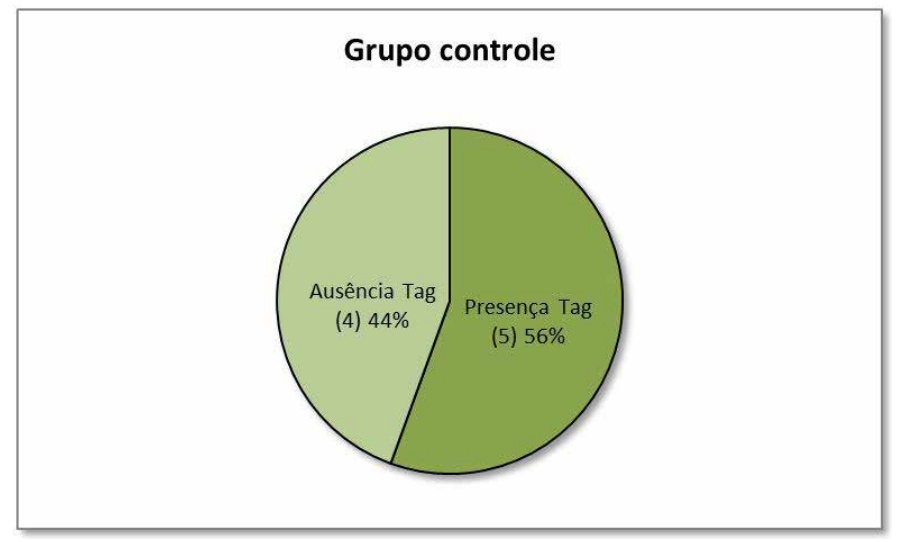

Fonte: dos autores

Figura 3 - Porcentagem de amostras com presença ou ausência de tags do grupo espatulado.

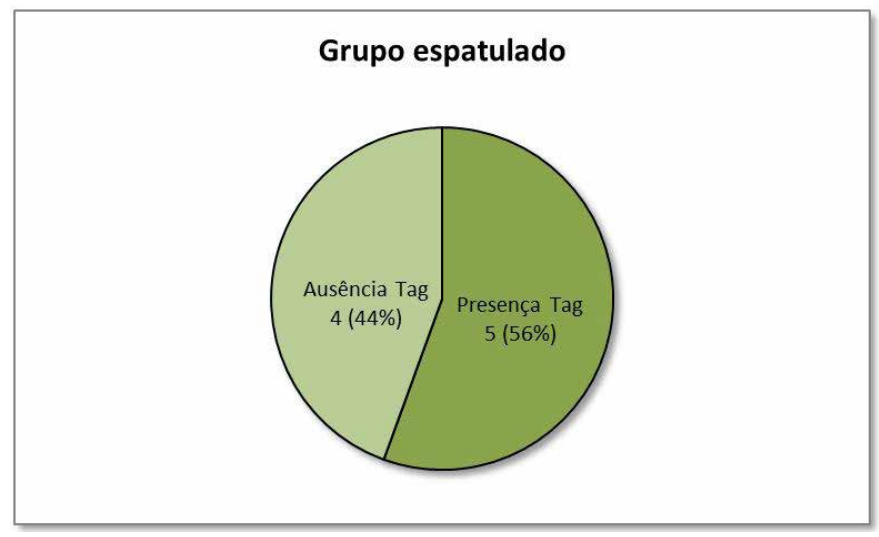

Fonte: dos autores 
Avaliação da presença de tags intratubular na região apical de dentes anteriores unirradiculares, variando a técnica de manipulação do cimento resilon

Figura 4 - A) Fotomicrografias (MEV) das amostras do grupo espatulado; B) Fotomicrografias (MEV) das amostras do grupo controle.
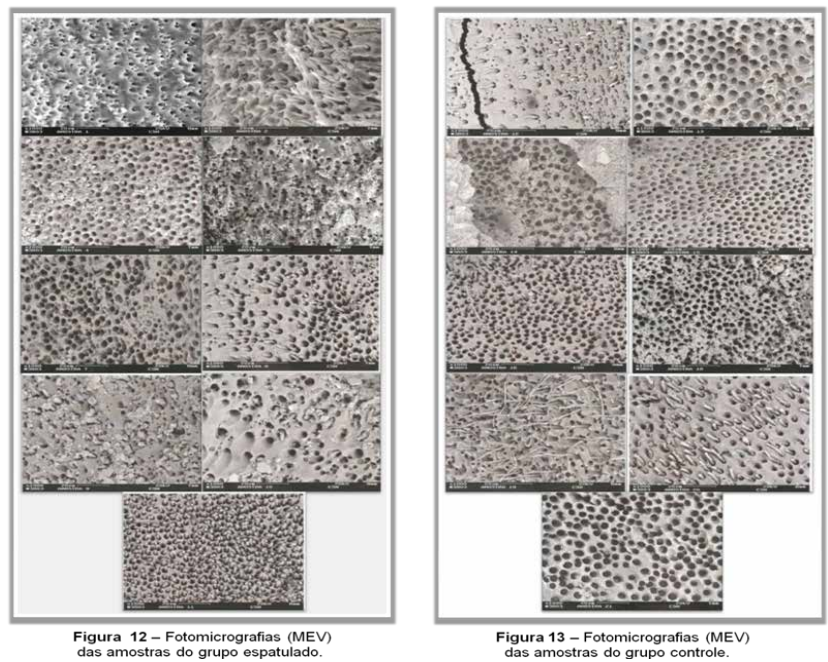

Fonte: dos autores

\section{DISCUSSÃO}

Atualmente, o sucesso do tratamento endodôntico está focado em princípios mais amplos, incluindo diagnóstico e plano de tratamento, conhecimento de anatomia, além dos conceitos tradicionais de debridamento, desinfeç̧ão e obturação. Portanto, a obturação tridimensional do espaço radicular é essencial para o sucesso em longo prazo, devendo o sistema de canais ser selado apical, coronal e lateralmente, favorecendo o reparo dos tecido periapicais (SCHILDER, 1967; JOHNSON; GUTMANN, 2010).

Sen et al. (1996) não acharam uma correlação entre a penetração do cimento e microinfiltração, utilizando a técnica de obturação de cone único. Esse resultado pode ser explicado pela ausência de força de compactação durante o procedimento clínico ou pela diferença de metodologia ou ferramenta de avaliação. Um ponto negativo visto na formação de tags é, se houver necessidade de retratamento, pode haver maior dificuldade de removê-los do interior dos túbulos dentinários, fato que pode interferir de maneira negativa na eliminação de microorganismos resistentes que podem fazer desse resíduo um substrato para seu crescimento (PIRANI et al., 2009).

Outro ponto a ser destacado refere-se à estrutura irregular e variável da dentina na região apical associada a um difícil controle da umidade, o que poderia prejudicar a penetração de adesivos para dentro dos túbulos dentinários e a formação da camada híbrida (MJOR et al., 2001). Ainda, um fator que pode interferir na polimerização do material obturador é a presença de oxigênio na região apical, impedindo a polimerização do cimento nessa região (XI WEI et al., 2010; LIMA et al., 2007). Segundo Guttman (1993) e Vassiliadis et al. (2004), o cimento tende a penetrar menos nos túbulos dentinários localizados mais apicalmente, devido a uma menor concentração de túbulos dentinários e menor diâmetro dos mesmos nessa região, além de receber uma menor força de compactação durante a obturação na maioria das técnicas de obturação. Baseados em tais estudos, justifica-se a escolha do terço apical para avaliação no presente trabalho, com o objetivo de suprir as deficiências da região, por ser a menos permeável e a mais crítica para todas as fases do tratamento endodôntico. 
Devido à boa adesão dentinária da dentística restauradora, pensou-se nesse sistema de adesão com formação da camada híbrida para cimentos endodônticos. A ideia do uso de cimentos à base de resina epóxica ou metacrilato como material obturador vem sendo enfatizada no mercado odontológico, sendo,primeiramente, estudada por Alberg \& Tay (1998), que propuseram uma mudança estrutural no tamanho das esferas da resina de um cimento ósseo ortopédico à base de metacrilato. Em 2004, foi lançado no mercado e apontado como possuidor de ótimas propriedades seladoras, o cimento endodôntico à base de resina metacrilato Resilon/Epiphany ${ }^{\circledR}$, sendo o cone de resilon e o cimento do mesmo material, formando-se um monobloco do material obturador com ótima adaptação à parede do canal radicular, adicionando o primer (SAKRI et al.,2010). Essa proposta tem como ideal a obtenção da adesão dentinária semelhante à da dentística restauradora, na qual ocorre grande penetração do adesivo no interior do túbulo dentinário (PATEL et al., 2007) com formação de inúmeros tags (FONTANA, 2007; HAMMAD et al., 2009; ORDINOLA- ZAPATA et al., 2009; DE DEUS et al., 2011; PUNITHA et al., 2011; RAVI et al., 2014). Mesmo comparando a penetração intratubular de outros cimentos, Sen et al. (1996) e Oksan et al. (1993) constataram que os cimentos resinosos, dentre outros, apresentaram os melhores índices de profundidade. Uma explicação para essa situação de vantagem, frente a cimentos à base de hidróxido de cálcio ou à base de óxido de zinco e eugenol, segundo Weis et al. (2004), seria que os resinosos possuem uma melhor integridade da matriz do cimento, após mistura ou espatulação. 0 tamanho das partículas formadas seria menor e, portanto, facilitaria seu escoamento para o interior dos túbulos dentinários, o que é mais difícil de ocorrer com os outros cimentos.

O cimento Epiphany ${ }^{\circledR}$ apresenta forma pasta/pasta acondicionadas separadamente em cartuchos de automistura. Quando o êmbolo é acionado, as pastas passam por espirais e saem com o cimento pronto para uso. A partir da observação de que o cimento dispersado pelo dispositivo "automix" não possuir aspecto visual homogêneo, com traços das diferentes pastas na sua massa, propôs-se nesse estudo a espatulação das referidas pastas. Haragushiku (2007) também propôs em seu estudo espatular o cimento, a fim de deixá-lo mais homogêneo, e verificou que, quando as paredes dentinárias foram tratadas com laser, o grupo do Epiphany ${ }^{\circledR}$ espatulado apresentou melhores resultados de tensão de cisalhamento e adesão. Segundo o autor, isso ocorreu provavelmente porque o laser promoveu irregularidades na superfície dentinária preenchidas pelo cimento espatulado, devido a seu maior escoamento em decorrência da homogeneização da mistura.

As pontas "automix" são fabricadas em um mesmo padrão de comprimento e largura, e quantidade de aspirais, não só para o cimento Epiphany ${ }^{\circledR}$, mas também, para outros cimentos. Junto a isso, após a colocação do cimento na placa de vidro, é possível visualizar traços das diferentes pastas na sua massa com aspecto não homogêneo. Devido a esse conjunto de fatores, este trabalho avaliou se a mistura acrescida desse cimento com espatulação manual por 10 segundos melhoraria a sua capacidade de formação de tags nos túbulos dentinários.

Tay et al. (2005) apontaram ainda a contradição entre o que é proposto pelo criador da resina e o que é instruído pelo fabricante do produto. Assim, ao propor um selador que se polimeriza em 45 minutos à temperatura ambiente, o criador do cimento endodôntico Epiphany ${ }^{\circledR}$ levou em consideração que a lenta polimerização dos seladores de resina poderá, via escoamento, melhorar, em razão do prolongado tempo de geleificação, concedendo uma chance de alívio para o stress da contração de polimerização. Segundo os autores, para criar um selamento coronário imediato, via polimerização com luz do selador de resina, as instruções escondem os benefícios derivados da lenta autopolimerização proposta para o cimento.

No presente estudo, não houve uma seleção da amostra do mesmo grupo dentário, podendo tal condição influenciar no resultado, já que no estudo realizado por Ribeiro et al. (2010) concluiu-se que a penetração de corante nos túbulos dentinários da região apical é extremamente dependente do 
Avaliação da presença de tags intratubular na região apical de dentes anteriores unirradiculares, variando a técnica de manipulação do cimento resilon

grupo de dentes avaliados. Outros fatores que podem influenciar a penetração de cimento em túbulos dentinários são: a técnica de obturação empregada, o ângulo entre a parede dentinária e o túbulo, o diâmetro do túbulo e o tipo de cimento endodôntico (KOUVAS et al., 1998).

Contudo, o cimento Epiphany ${ }^{\circledR}$ poderá ser uma alternativa de cimento endodôntico que oferece adesão na dentina radicular com formação de tags no terço apical, porém, mais estudos de evolução clínica devemavaliar antes de ser usado rotineiramente na prática endodôntica.

\section{CONCLUSÃO}

Conclui-se, frente à metodologia utilizada, que os grupos tiveram o mesmo perfil de presença de tags produzidos, quando acrescida a espatulação manual do cimento preparado com a ponta "automix". Portanto, não é necessário espatular, simplificando e agilizando a técnica obturadora, obedecendo a orientação do fabricante.

\section{REFERÊNCIAS}

AHLBERG, K. M. F.;TAY W. M.A. Methacrylate cement used as a root canal sealer. International Endodontic Journal, v. 31, p. 15-21, 1998.

APTEKAR, A.; GINNAN, K. Comparative analysis of microleakage and seal for 2 obturation materials: Resilon/Epiphany and gutta-percha. J Can Dent Assoc, vol. 72, p. 245, 2006.

DE DEUS J.; REIS C.; GIORGI K. D.; BRANDÃO M. C.; AUDI C.; FIDEL R. A. S. Interfacial adaptation of the Epiphany self-adhesive sealer to root dentin. Oral Surgery, Oral Medical, Oral Pathology, Oral Radiology and Endodontic. v. 111, p. 381-6, 2011.

Fontana CE. Avaliação "in vitro" da penetração intratubular de diferentes cimentos endodônticos. 2007. Dissertação (Mestrado em Odontologia, área de concentração endodontia) - CPO São Leopoldo Mandic, Campinas, 2007.

GHARIB S. R.;TORDIK P. A.; Imamura G. M.;Baginski T. A.; Goodell G. G. A confocal laser scanning microscope investigation of the Epiphany obturationsystem. Journal Endodontic. v. 33, p. 957-61, 2007.

GUTMANN JL. Adaptation of injected thermoplasticized gutta-percha in the absence of the dentinal smear layer. International Endodontic Journal. v. 26, p. 87-92, 1993.

Hammad M.; Qualtrough A.;Silikas N. Evaluation of root canal obturation: a three-dimensional in vitro study. Journal of Endodontic. v. 35, p.541-544, 2009.

HARAGUSHIKU G. A. Avaliação do efeito de diferentes tratamentos da superfície dentinária sobre a resistência de união à dentina de cimentos endodônticos. 2007. Dissertação (Mestrado em Odontologia, área de concentração endodontia) - Universidade de Ribeirão Preto, RibeirãoPreto, 2007.

IMAI Y.; KOMABAYASHI T. Properties of a new injectable type of root canal filling resin with adhesiveness to dentin. Journal of Endodontic. v. 29, p.20-23, 2003. 
JOHNSON W. T.; GUTMANN J. L. Obturação do sistema de canais radiculares limpos e modelados. In: Caminhos da polpa. 9a edição; Rio de Janeiro: editora Guanabara Koogan, 2010. P. 358-399.

KOUVAS V.; LIOLIOS E.; VASSILIADIS L.; PARISSIS-MESSIMERIS S.; BOUTSIOUKIS A. Influence of smear layer on depth of penetration of three endodontic sealers: an SEM study. Endodontic Dental Traumatology, v. 14, p. 191-5, 1998.

LIMA R. M. N.; NUNES E.; SILVEIRA F. F. Comparação do selamento apical após obturação dos canais com os sistemas guta-percha / $\mathrm{AH} 26{ }^{\circledR}$ e Resilon ${ }^{\circledR} /$ Epiphany ${ }^{\circledR}$. Revista da Faculdade de Odontologia. v. 12, p. 56-60, 2007.

MJOR I. A.; SMITH M. R.; FERRARI M.;MANNOCCIF. The structure of dentine in the apical region of human teeth. International Endodontic Journal. v. 34, p.346-53, 2001.

Oksan T.; Aktener B. O, Sen BH et al.The penetration of root canal sealer into dentinal tubules.Ascanning. Electron microscopic study. International Endodontic Journal. v. 26, p. 301-5, 1993.

ORDINOLA-ZAPATA R.; BRAMANTE C. M.; BERNARDINELI N.; GRAEFF M. S.; GARCIA B. B.; MORAES I. G.; DEBELIAN G. A. Preliminary study of the percentage of sealer prenetration in roots obturated with the TermaFil and RealSeal-1obturation techniques in mesial roots canals of mandibular molars. Oral Surgery, Oral Medical, Oral Pathology, Oral Radiology and Endodontic. v. 108, p. 961-8, 2009.

PATEL D. V.; SHERRIFF M.; FORD T. R. P.; WATSON T. F.;MANNOCCI F. The penetration of Real Seal primer and Tubliseal into root canal dentinal tubules: a confocal microscopic study. International Endodontic Journal. v. 40, p. 67-71, 2007.

PIRANI C.;PELLICCIONI G. A.; MARCHIONNI S.;MONTEBUGNOLI L.;PIANA G.;PRATI C. Effectiveness of three different retreatment techniques in canals filled with compacted gutta-percha or Thermafil: a scanning electron microscope study. Journal Endodontic. v. 35, p.1433-40, 2009.

PUNITHA P. G.; SHASHIKALA K. Evaluation of the adaption of resin based sealers Epiphany, AH Plus and $\mathrm{AH} 26$ to the root canal dentin by scanning electron microscope. Indian Journal Stomatology, v. 4, p. 207-211, 2011.

RAVI S. V.;NAGESWAR R.;SWAPNA H.;SREEKANT P.;RANJITH M.;SURABHI M. Epiphany sealer penetration into dentinal tubules: confocal laser scanning microscopic study. Journal of Conservative Dentistry, v. 17, p. 179-82, 2014.

RIBEIRO R. G.; MARCHESAN M. A.; SILVA R. G.; SOUZA-NETO M. D.; PÉCORA J. D. Dentin permeability of the apical third in differnt groups of theeth, Brazilian Dental Journal, v. 21, p. 216-9, 2010.

SAKRI M. R.; SHELLY D.;HINDHUJA D.; BYAKOD P.; NAGESWAR R. Resilon - "Endobondics". Pravara Medical Review, v. 2, p. 4-9, 2010.

SALEH I. M.; RUYTER I. E.; HAAPASALO M.; ORSTAVIK D. Survival of Enterococcus faecalis in infected dentinal tubules after root canal filling with different root canal sealer in vitro. International Endodontic Journal, v. 37, p.193-198, 2004.

SCHILDER H. Filling root canals in three dimensions. Dental Clinics of North America. v. 11, p. 723-44, 1967. 
Avaliação da presença de tags intratubular na região apical de dentes anteriores unirradiculares, variando a técnica de manipulação do cimento resilon

SEN B. H.;PISKIN B.;BARAN N.The effect of tubular penetration of root canal sealer on dye microleakage. International Endodontic Journal, v. 29, p. 23-8, 1996.

SIQUEIRA JR, J. F; RÔÇAS I. N.; FAVIERI A.; ABAD E. C.; CASTRO A. J. R.; GAHYVA S. M. Bacterial leakage in coronally unsealed root canals obturated with 3 different techniques. Oral Surgery, Oral Medical, Oral Pathology, Oral Radiology and Endodontic, v. 90, p. 647-650, 2000.

SHIPPER G.; ORSTAVIK D.; TEIXEIRA F. B. et al. An evaluation of microbial leakage in roots filled with a thermoplastic synthetic polymer-based root canal filling material (Resilon). Journal Endodontic, v. 30, p. 342-7, 2004.

TAY F. R.;LOUSHINE R. J.; WELLER N.; KIMBROUGH W. F.; PASHLEY D. H.;MAKYIU-FAI; LAI C. S.; RAINA R.; WILLIAMS M. C. Ultrastructural evaluation of the apical seal in roots filled with a polycaprolactonebased root canal filling material. Journal Endodontic, v. 31, p. 514-9, 2005.

VASSILIADIS L. P.; SKLAVOUNOS S. A.; STAVRIANOS C. K. Depth of penetration and appearance of Grossman sealer in the dentinal tubules: an in vivo study. Journal Endodontic, v. 20, p. 373-6, 1994.

XI WEI D. S.; JUN-QI LING W. W. Resilon: a methacrylate resin-based obturation system. J Dent Sci. v. 5, p. 47-52, 2010.

WEISS M. V.; PARASHOS P.; MESSER H. H. Effect of obturation technique on sealer cement thickness and dentinal tubule penetration. International Endodontic Journal. v. 37, p. 653-63, 2004.

ZIDAN O.;ELDEEB M. E.The use of a dentinal bonding agent as a root canal sealer. Journal of Endodontic. v. 11, p. 176-8, 1985. 